\title{
Circuit
}

Musiques contemporaines

\section{Une " pièce habitable " : Watt de Pascal Dusapin}

\section{Igor Ballereau}

Volume 29, numéro 1, 2019

Pascal Dusapin : la parallaxe des voix

URI : https://id.erudit.org/iderudit/1059428ar

DOI : https://doi.org/10.7202/1059428ar

Aller au sommaire du numéro

Éditeur(s)

Circuit, musiques contemporaines

ISSN

1183-1693 (imprimé)

1488-9692 (numérique)

Découvrir la revue

Citer ce document

Ballereau, I. (2019). Une " pièce habitable ": Watt de Pascal Dusapin. Circuit, 29(1), 63-67. https://doi.org/10.7202/1059428ar d'utilisation que vous pouvez consulter en ligne.

https://apropos.erudit.org/fr/usagers/politique-dutilisation/ 
DOCUMENTS

\section{Une «pièce habitable » : Watt de Pascal Dusapin}

Igor Ballereau

En 1992, Igor Ballereau rencontrait Pascal Dusapin pour la première fois. Cette rencontre fut un choc important, incitant Ballereau à devenir lui-même compositeur. De l'automne 2014 à l'automne 2016, il a rédigé un livre encore inédit, intitulé Les pièces habitables, constitué de 122 essais aux tonalités libres, volontiers littéraires, et qualifiés par l'auteur de «visites à la musique de Pascal Dusapin ». Nous publions ici un extrait de ce livre, dans lequel il est question du concerto pour trombone Watt (1994). Rappelons que le soliste de cette œeuvre, commandée par le Nevada Symphony Orchestra, était le tromboniste québécois Alain Trudel, qui a joué un grand rôle dans le répertoire pour trombone de Dusapin.

\section{Watt (extrait du chapitre 22, "Voix gigognes")}

Ce n'est pas toujours le cas, loin de là en fait, mais il arrive qu'une œuvre musicale porte en son sein la troublante puissance d'une question sans réponse. «Difficile d'accès", dira-t-on peut-être - pour rester poli - après l'avoir écoutée. Et d'un certain point de vue on ne croira pas si bien dire: le temps affecté y prend la forme d'un embrouillamini où plus rien ne commence ni ne finit vraiment. À sa source, la question - ou plutôt l'énigme, tant la musique l'exprime sans l'énoncer - comprime fortement un endroit singulier du temps, et sa radiation est telle depuis cet endroit que le temps s'en trouve rendu concentrique et omnidirectionnel. Il acquiert un milieu à l'entour duquel il se problématise telle une nappe froissée, recevant l'expansion de coïncidences volontiers insolites qui vont s'interconnecter en tous sens. Il y a de quoi s'y perdre, et je veux dire: bien!, et je veux dire: tant mieux!, car c'est la chance offerte d'y inventer son chemin. D’autant plus que la périphérie labyrinthique des coïncidences dépasse de loin l'enveloppe audible de la 
musique. Silence d'avant comme silence d'après, silence autour en somme, l'étendent à la multiplicité des formes du monde où l'effet de constellation se propage sans arrêt.

Au milieu (tel que je le conçois) de l'œuvre symphonique de Charles Ives, riche de plages événementielles qui s'interpénètrent tout en tournoyant librement les unes par rapport aux autres, rayonne une petite pièce d'allure presque simplette, intitulée The Unanswered Question. Alentour (avec pour apogée la Symphony No. 4) : hymnes, berceuses, danses, ballades - eux-mêmes parcourus de rivières, de poètes, de soirs, d'armées en parade, etc. - ne cessent d'entrer en collision dans un crépitement d'éclairs digne de la Genèse (un comble, soit dit en passant, pour Ives qui gagnait sa vie comme assureur). Mais voilà: le milieu influent d'un morceau de musique donné ne se trouve pas forcément en son milieu, soit la moitié de sa durée (quoiqu'il le puisse), ni même en un point quelconque de sa durée (quoiqu'il le puisse). Il peut aussi se tenir ailleurs, agir à distance depuis une autre œuvre, ne lui ressemblant peut-être pas du tout, déjà faite ou encore à faire, voire enfin ni l'un ni l'autre: certaines vies créatrices sillonnent inlassablement la périphérie d'une question dont elles ne découvriront jamais la source irradiante; ou bien alors ce sera que, l'ayant jaugée de loin, elles auront préféré l'abandonner au silence total.

De toute façon, le milieu de l'œuvre d'un compositeur $x$ ou $y$ ne se laissera pas localiser une fois pour toutes, du moins pas tant que ce dernier vit. Car il s'excentre en permanence, comme déporté par l'expansion asymétrique, les bords toujours changeants de sa périphérie. Dût-il se manifester, apparaître à tel moment précis de telle pièce, on n'aura pas pour autant ruiné la possibilité de le voir réapparaître en tel autre point de telle autre pièce, et ce de plus sous une forme probablement inédite. Le milieu n'est pas seulement mobile, il est polymorphe; sans doute même ne possède-t-il pas de figure en soi : son aspect découlera chaque fois, bien qu'en le renversant, de celui de la région périphérique par laquelle il s’atteint.

Et sur ce plan-là, comme du reste la musique d'Alban Berg dont elle est consanguine, la musique de Pascal Dusapin nous gâte, car le centre névralgique de sa question sans réponse y est aussi puissant que son apparition est fréquente et, surtout, sa physionomie variable. Correspond-il à l'onde bruiteuse au beau milieu de Quad? Au brouillard frémissant, a punta d'arco du Quatuor V? Au contrepoint austère des six cors à l'ouverture d'Uncut? À la mélopée conjonctive qui traverse la «Zone 13» du Quatuor II? Au cri en forme de cime par lequel se termine If? Au trou de silence par lequel se régénère To God? Celui par lequel se montre Khôra? J'en passe. A priori, rien ne permet de rapporter cette pluralité d'apparences à un seul et même objet. 
Et cependant, derrière chacune d'elles hurle son immanquable signature: le temps se fige, la transmission des coïncidences ne se fait pas ou plus, la forme est stupéfiée.

Par contraste avec la périphérie qu'il dynamise en y provoquant des événements à foison, le milieu lui-même est un peu comme l'œil d'un cyclone, une sorte de négatif de ce qui l'entoure. En lui, la situation se renverse du tout au tout. Une singularité absolue assujettit le temps à sa présence. L'effet de constellation, la dissémination des éclairs cèdent la place à une force suspensive. On entre dans l'éternité, pour ainsi dire localement. Et quand le silence ne l'emporte pas tout à fait, la couleur instrumentale qui subsiste gèle, comme arrêtée à son contact. C'est un moment à la beauté extraordinaire. Le matériau musical n'y est pour rien. Il est alors ramené à une telle simplicité qu'on serait tenté de dire: «Moi aussi, je peux faire ça.» Mais il serait malhonnête ou erroné de l'extraire de son contexte, de l'isoler de ce qui lui arrive à ce moment-là, ce justement par quoi il se trouve ainsi déparé: l'emprise infinie d'une question asymptote à sa réponse, toujours s'en rapprochant sans jamais la rencontrer.

Alors j'en viens enfin à Watt; car si, chez Pascal Dusapin, la formidable étreinte du milieu ne paie souvent pas de mine, elle y produit cette fois une forme qui n'était pas de ce monde jusque-là. Quant à la beauté de cette forme, je serais bien en mal d'en parler. Dire la beauté est très difficile. Il faudrait une syntaxe, voire, en certains cas, des mots nouveaux. Comme l'effroi, la beauté coïncide avec un état limite du langage : à leur rencontre il doit sérieusement se dépasser ou s'évanouir. C'est la merveilleuse affaire des poètes, et je n'en suis pas un. Je veux néanmoins tenter de décrire la voix composite que se découvre Watt à mi-chemin de son parcours, surtout parce qu'elle fonde à elle seule le concept de voix gigogne. Et par «voix», j'entends une fois de plus «corps». Bien sûr, en général, on aura raison de distinguer la première du second comme étant l'une de ses propriétés parmi d'autres, mais en musique, j’insiste, c'est tout un: le corps affirme sa nature sonore; non pas son qu'il produit à l'occasion, pour communiquer par exemple, mais son qu'il est à chaque instant et qui se donne à percevoir pour peu qu'on en monte le volume.

J'appelle voix gigogne toute voix $v$ se prolongeant sans cesser de renvoyer à un seul et même individu par une autre voix $v^{\prime}$, qualitativement différente, voire plus loin par une autre $v$ ", et même (et là, c'est dingue) encore par une autre $v$ "' (dans le cas de Watt).

On a vu, avec l'exemple éloquent d'Itou, que v' pouvait émerger du spectre harmonique discrètement supporté par une fondamentale $v$, soit au moyen 
1. Variante: "C'est ce que je fais qui m’apprend ce que je cherche"; Pierre Soulages, inlassablement cité par Dusapin.

2. Henri Michaux (1963), "Lointain intérieur", in Plume, précédé de Lointain intérieur, Paris, Gallimard, p. 113. d'un instrument unique. Puis on a vu, avec l'exemple non moins éloquent de Laps, que $v^{\prime}$ pouvait en outre émerger au moyen d'un second instrument, distinct de celui ayant posé $v$, et semblant coulisser hors de lui. Surtout on aura vu, dans un cas comme dans l'autre, que l'extension de $v$ en $v$ ', loin de se résumer à une bidouille d'instrumentation, correspondait à une transition de phase pour la musique elle-même : altération de l'identité couronnant une quête aussi obsédante qu'épuisante pour Itou; passage de la plainte irrecevable en zone profonde pour Laps.

Or, pour Watt, parler de «transition de phase» serait un euphémisme. Disons donc les choses autrement: un morceau de musique tellement paumé qu'il paraît devoir concentrer sur son seul corps toute la vigueur rizhomatique de l'œuvre à laquelle il se rattache tombe soudain, comme à la renverse, sur le foyer de sa question sans réponse, pile au milieu de cette même œuvre. Il ne l'a pas vu venir, même s'il le cherchait comme un fou («Mais tu cherches quoi? - Je ne sais pas... je le saurai quand je l'aurai trouvél»), ni nous non plus d'ailleurs, qui le suivions tant bien que mal des oreilles. Il faut l'entendre pour le croire: en quelques secondes, la musique bascule d'un terrain compliqué, meuble, accidenté, fouetté de vents contraires et de giboulées... à la pure et simple douceur ascendante d'une colonne d'air.

Tout à coup, grand bonheur, une phrase... un incident... un je ne sais quoi, il y a là quelque chose... Alors il se met à léviter vers ce quelque chose avec le plus qu'il peut de lui-même, parfois s'y accole d'un coup comme le fer à l'aimant. Il y appelle ses autres notions: «venez, venez». Il est là quelque temps dans les tourbillons et les serpentins et dans une clarté qui dit «c'est là».

C'est là... mais justement, là, qu'advient-il de Watt? Quel impact physique? Que devient cette voix-corps-individu jusque-là assimilée à la sonorité normale du trombone, et que la distorsion par quelques sourdines n'avait pas touchée au cœur? Écoutez bien... Watt se découvre un être télescopique! Suivez-le bien: le son grave $v$, joué normalement, s'augmente du son $v$ ', fredonné plus haut dans l'instrument, lesquels combinés s'étendent toujours plus haut au son harmonique $v$ ", lui-même annexant un instrument supplémentaire, le piccolo, pour s'étirer encore à l'octave supérieure, en $v$ ". De la base $v$ au sommet $v$ "', la chose déployée atteint par instants quarante-trois demi-tons chromatiques de haut! C'est un peu comme si basse, ténor, alto et soprano chantaient d'une seule voix en un même corps. Un corps qui s'étend de la bête à l'ange, une baleine quadriphonique. Et ce corps a lieu comme une scène verticale : tout en bas, tandis que le bourdon $(v)$ s'allonge et se fond parmi les violoncelles de l'orchestre, le fredon $\left(v^{\prime}\right) s^{\prime}$ en extrait en le caressant. De leurs amours fréquentielles naît une sorte d'olifant ( $v$ "), timbre où l'on 
ne sait départager la détresse de la volupté. Juché sur les deux premières composantes de la voix qui s'effacent pour le laisser paraître, l'olifant ressort encore davantage par son expressivité charnelle; on pourrait le toucher, on voudrait le prendre dans ses bras. À travers lui, Watt ressemble vraiment à tout le monde: une modeste agrégation d'atomes courtisée par la mort. Mais l'olifant n'est pas le bout de la voix, pas plus qu'il n'est l'ultime secret du corps ainsi déboîté. À distance d'une octave plus aiguë, au niveau de la première harmonique de l'olifant (lui-même déjà une harmonique), vibre pour finir le sopranino ( $v$ "'). Harmonique de l'harmonique, l'extrémité de la voix gigogne est un «mignon dessus», une pointe de cristal, un diamant effilé qui touche les microsillons cosmiques; finesse, limpidité, avec un je ne sais quoi d'indolent dans la froideur du timbre. Et si c'en est alors la découverte par Watt, ce n'est pas la dernière fois que Pascal Dusapin verra son piccolo chanter avec un tel calme (lui si bien doté pour la vitesse et l'éclat), loin à l'écart des passions. On le retrouvera dans Perelà, Uomo di fumo, mais cette fois plus attaché à rien, éjecté de ses étages «terrestres» et sondant tout seul comme un grand le silence de la question sans réponse.

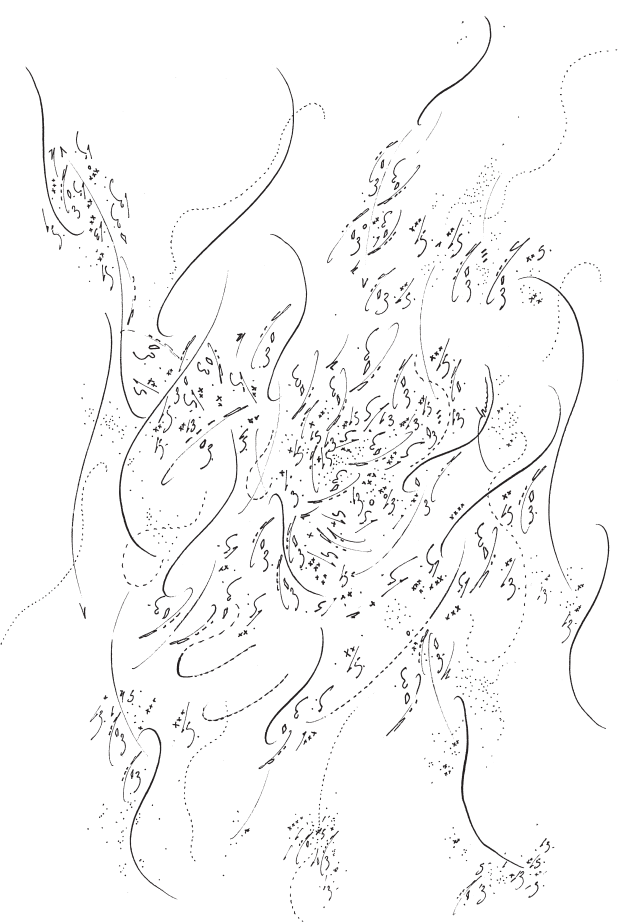

Pascal Dusapin, esquisse pour Mille Plateaux, 2014. Encre sur papier, $27 \times 29 \mathrm{~cm}$. 